\title{
EL CIMARRÓN Y LAS CONSECUENCIAS DE LA GUERRA DEL 95. UN REPASO DE LA BIOGRAFÍA DE ESTEBAN MONTEJO
}

POR

\author{
MICHAEL ZEUSKE
}

Universidad de Colonia, Alemania

A través del analísis de documentos archivísticos (protocolos notariales) y periódicos del año 1912 se ofrece una lectura crítica de la novela-testimonio Biografia de un Cimarrón (1966), de Miguel Barnet, y se discuten los resultados de la Guerra de Independencia cubana (1895-1898), comparando las perspectivas literarias de Barnet, la narración de Montejo y el discurso de las fuentes documentales.

"Aunque por supuesto nuestro trabajo no es histórico" (Miguel Barnet)

Aparte de El Ingenio de Manuel Moreno Fraginals, no existe apenas ningún libro en la historiografía cubana que se haya difundido de forma tan extensa como la Biografía de un Cimarron de Miguel Barnet. Tanto en el debate actual sobre la esclavitud como en el género de las memorias históricas sobre esclavos en general, y no sólo en las cubanas, es a pesar de una serie de contradicciones, el autor clásico por excelencia del testimonio literario. Esto lo demuestran, en

ABREVIATURAS UTILIZADAS:

ANC: Archivo Nacional de Cuba (La Habana)

APC: Archivo Provincial de Cienfuegos (Cienfuegos)

USNA: United States National Archives (Washington, D.C.) 
último término, no sólo las nuevas ediciones ${ }^{1}$ y traducciones, como las que se recogen con el título Biography of a Runaway Slave en los Estados Unidos de América y en Gran Bretaña ${ }^{2}$, sino también en los debates desencadenados por el libro de Barnet ${ }^{3}$.

La primera edición de ambos libros, El Ingenio 4 y el Cimarrón ${ }^{5}$ se publicó, aproximadamente, a mediados de los sesenta. Ambos re-

1 El libro se edita en 1995, en Alemania, ya en la séptima edición con el título: Der Cimarrón. Die Lebensgeschichte eines entflohenen Negersklaven aus Kuba von ihm selbst erzählt. Nach Tonbandaufnahmen herausgegeben von Miguel BARNET, übersetzt von Hildegard BAUMGART, mit einem Nachwort von Heinz Rudolf SONNTAG und Alfredo CHACON, Frankfurt am Main, Editorial Suhrkamp 1995,1 ${ }^{\text {a }}$ edición: 1976; licencia de la editorial Insel-Verlag. Mis agradecimientos a Rebecca J. Scott University of Michigan, por haberme propuesto el publicar este artículo, a los colegas del coloquio sobre "Race and Politics at the turn of the Century: Cuba, 1895-1917" de la New York University, a Ada Ferrer y Alejandro de la Fuente. Agradezco sobre todo a Grisel Ramos Maceda (Santa Clara) y a Orlando García Martínez (Archivo Provincial de Cienfuegos) con sus colaboradoras, así como a Martin Franzbach (Universität Bremen), a Magnus Mörner (Suecia), a Karin Schüller (Universität zu Köln) y a Matthias Perl (Universität Mainz-Germersheim) por las indicaciones, búsqueda conjunta en los archivos, discusiones y lectura crítica. El artículo presente es una versión revisada del artículo en inglés, publicado primero en New West Indian Guide/Nieuwe West-Indische Gids, vol. 71, nums. 3 \& 4, 1997, pp. 265-279 Leiden, Baises Bajos, KITLV Press. Las investigaciones de campo para este artículo fueron financiadas por la Sociedad Alemana de Investigación (DFG), como parte del proyecto: La transformación de la expansión europea (siglos XVI-XX).

2 The Autobiography of a Runaway Slave, ed. BARNET; translated from the Spanish by Jocasta INNES, New York, Pantheon Books 1968; The Autobiography of a Runaway Slave, Esteban MONTEJO, BARNET, Introd. and Bibliographical Essay: Alistair HENNESSY, London 1993 (utilizando la traducción anteriormente mencionada); Biography of a Runaway Slave, ed. by BARNET, translated by Nick HILl, Curbstone Press, East Haven, CT 1994.

3 Citamos solamente un ejemplo alemán: Harald WeNTZLAFF-EgGEBERT, Harald, Miguel Barnet's 'novela-testimonio' Biografia de un cimarrón: Life Story of a Runaway Slave, Ethnological Study, or Manipulation of Public Opinion en Slavery in the Americas, ed. by Wolfgang BINDER, Würzburg, Verlag Königshausen \& Neumann GmbH 1993, pp. 627-647. Ver también la breve selección bibliografica en el anexo.

4 Manuel Moreno Fraginals, El Ingenio, Comisión Nacional Cubana de la UNESCO, La Habana 1964.

5 BARNET, Biografia de un cimarrón, La Habana: Instituto de Etnología y Folklore 1966. A decir verdad, el mismo Barnet comenzó los trabajos con el Cimarrón como un complemento de las investigaciones sobre las condiciones en las que vivían los esclavos de las plantaciones, según la representación que Juan PÉREZ DE LA RIVA, "El barracón de ingenio en la época esclavista", en: El barracón y otros ensayos, La Habana 1975, pp. 1574 hizo de las mismas, aunque a nuestro parecer el testimonio de Montejo se puede considerar como complemento adicional del de Moreno Fraginals, incluso si se tiene en cuenta que éste último sólo llega en el tiempo, hasta mediados de los sesenta del siglo XIX.; veáse: BARNET, Para llegar a Esteban MONTEJo: "Los caminos del Cimarrón", en Contracorriente. Una revista cubana de pensamiento, Oct./Nov./Dic. 1996, año 2, núm. 6, pp. 29-

R. I., $1998, \mathrm{n}^{\circ} 212$ 
presentan, algo así, como a los dos gemelos, aunque completamente distintos, de la historiografía revolucionaria cubana; los dos nacen al mismo tiempo y alcanzan rápidamente fama y prestigio.

Moreno escribió un análisis marxista sobre la historia estructural y social del complejo núcleo de la producción del azúcar según los requisitos establecidos por la esclavitud. Por el contrario, la narración de Montejo y el estudio de Barnet, recogen de forma individual, por así decirlo, la perspectiva interna de esta forma social de opresión, precisamente no desde el punto de vista de un esclavo de las plantaciones, sino justo desde el de un cimarrón, un ex-esclavo que se había liberado a sí mismo.

Un ex-esclavo centenario narra las estructuras harto opresivas y los deseos implacables de libertad de un afrocubano criollo esclavizado: ¿qué mejor símbolo se puede desear para una narración maestra de la temprana historiografía cubana?

Mientras tanto, ambos libros han entrado en años y han alcanzado la edad correspondiente a una generación histórica. Moreno continúa siendo considerado como el clásico de las estructuras, incluso cuando una serie de declaraciones suyas se falsificaran a través de exhaustivas investigaciones ${ }^{6}$ y su libro además "sólo" descubra el período de tiempo en el que tuvo lugar la esclavitud. Además hoy, las heróicas perspectivas de los años sesenta y comienzos de los setenta sobre la Historia de Cuba recaen en el desencanto, la desilusión y la revisión.

¿Para qué leer entonces de nuevo el Cimarrón? Si bien el libro es un clásico, además de ser el libro de texto de escuelas y seminarios universitarios de introducción, en el transcurso de su vida en papel, fue criticado frecuentemente, sobre todo, por historiadores especializados. En contra de Barnet y de su Cimarrón se realizaron públicamente objeciones, bien de tipo metodológico o de contenido, así como en contra de los métodos de la forma "oral-history" que utilizaba, en interés de la literatura blanca, la mímesis fáctica con las palabras de un narrador negro en contra de los principios de cuestio-

44, pp. 30 y ss. Citamos aqui según la edición: M. BARNET, Cimarrón, La Habana: Gente Nueva, Instituto del Libro, 1967.

6 Sobre todo: Rebecca J. ScotT, Slave Emancipation in Cuba. The Transition to Free Labor, Princeton, New Jersey, Princeton University Press, 1985 y recientemente: Gloria GarCía, La esclavitud desde la esclavitud. La visión de los siervos, México Centro de Investigación "Ing. Jorge Y. Tamayo", 1996. 
namiento y selección de Miguel Barnet. Se censuró el relato, desde la perspectiva de la Cuba oficial de principios de los sesenta, la heroización, el antiimperalismo o la sexualización de la libertad a través de Montejo, su propia representación como individuo independiente que se suspende libremente en lo fáctico, sin ataduras sociales ni organizativas duraderas. La crítica del "gremio" fue exacerbada".

En relación con ésto, al autor de estas líneas se permite una breve recapitulación de sus propias lecturas sobre la biografía de Montejo. En 1970 se publicaba el Cimarrón, también en Berlin (este) ${ }^{8}$. La adquisición de la licencia por la editorial Insel Frankfurt am Main se había quedado con el libro - desde el punto de vista actual- un epílogo miserable y una máquina con fallos propios. Pero como quiera que fuese, la fantasía histórica ensilló a Pegaso y voló a los lugares místicos de Sagua la Grande, Cruces, Lajas y Palmira, se sentó en el balancín, al lado del narrador centenario o le acompañó en su huída a las montañas, rebelándose junto a él contra los dueños de esclavos. Mientras las páginas de los libros de texto históricos estaban, sólo de esta forma, plagadas de modelos, estructuras, clases y otros demonios amorfos, el texto-testimonio, aparentemente simple, presentaba a un individuo libre, en medio de la naturaleza tropical, deidades africanas todopoderosas y hombres y mujeres llenos de vida.

Montejo contaba cosas de la vida cotidiana, el juego de cartas, el comer y el beber, la sexualidad y la revolución, así como sobre la Guerra de la Independencia. Pero ante todo, se podía reconocer la voluntad, a la hora de afirmar la sabiduría de la vida y las singulares perspectivas de un ex-esclavo en una sociedad, en la que fueron tachados él y sus semejantes de "salvajes". En una palabra: ¡entusiasmo! Era como si los poderosos Orishas provenientes de la fantasía del texto, cogieran al lector y lo secuestraran en los mundos de Esteban Montejo.

7 Una de las críticas más objetivas en lengua alemana se encuentra en Martin FRANZBACH, "Entre la novela y el testimonio": Miguel BARNET en HANS-OTTO DILL et al., Apropiaciones de la realidad en la novela hispanoamericana de los siglos XIX y XX, Frankfurt a. M./Madrid, pp. 327-337. Desde una perspectiva sociolingüística crítica a la vez: Ulrich FLEISCHMANN, Ulrich, Miguel Barnet, etnógrafo y narrador en Historia y Grafia, Universidad Iberoamericana, núm. 2 (1994), pp. 125-141. En lengua inglesa ver la excelente introducción: HENNESSY, [2], pp. 1-23 (también aquí un buen ensayo bibliográfico de 1993).

8 Der Cimarrón ,[1]. 
Después, sin embargo, llegaron revisiones de la obra, en las que se les preguntó a otros. Magnus Mörner, en su visita a Leipzig en 1986, informó sobre los problemas relativos a historia-oral y el libro aterrizó en la estantería del historiador principiante, entre los libros de "ficción". El cimarrón parecía haber muerto auque todavía era válido "Habent sua fata libelli" y sobre todo: "Totgesagte leben länger" ("muerte anunciada, vida larga").

Como el autor pudo corroborar pronto, ya que a él le ocurrió lo mismo durante sus primeras estancias de investigación en Cuba, en 1987 y 1988 uno no quisiera, en modo alguno, perderse el relato. Tan pronto como los críticos más austeros trataron directamente con la historia social cubana de los siglos XIX y principios del XX, utilizaron el libro, lo leyeron para criticarlo de nuevo o para inspirarse, $o$ sencillamente para informarse sobre la visión particular de Montejo sobre determinados acontecimientos -acaso más de un lector o lectora-, sin citar el libro, porque, claro, entre los especialistas era considerado como algo informal.

En relación con las lecturas del Cimarrón, fue más bien una coincidencia, cuando se inició en 1993 un proyecto de la "Sociedad Alemana de Investigación" (DFG), en Cuba Venezuela y Brasil, sobre la integración de los antiguos esclavos en la cultura política del tiempo de la post-esclavitud. Barnet o su libro no fueron de ninguna manera la razón de la elección de Cuba, que en el Cimarrón también se describe como campo de acción de Esteban Montejo. La región cubana que elabora el proyecto es la zona del hinterland de Cienfuegos.

Esta clásica región azucarera que albergó, a finales del siglo $\mathrm{XIX}$, principios del XX, las centrales azucareras más grandes del mundo ${ }^{9}$, se convirtió tras la abolición de la esclavitud en Cuba (1886) y después de la guerra de la independencia contra España (1895-1898), en un centro de atracción para los libres trabajadores negros.

Desde 1993 hasta 1997, en el transcurso de las tareas relativas al proyecto, se examinaron, poniéndose al mismo tiempo de relieve, un sinfín de fuentes regionales. Además el Cimarrón de Miguel Barnet

\footnotetext{
9 Orlando GaRCía MARTíNEZ, Cienfuegos, Transformaciones en la industría azucarera entre 1878 y 1888. Los comerciantes y la implementación del central, Cienfuegos 1997 (material manuscrito, no publicado, lo utilizamos con el permiso del autor).
} 
se volvió a utilizar como lectura adicional, más bien como algo vergonzoso, justo de la misma forma que se ha expuesto anteriormente.

A pesar de todo, nosotros utilizamos el Cimarrón porque no hay o casi no existen testimonios objetivos de anteriores esclavos y por consiguiente, como posible perspectiva en cuanto al tiempo histórico (1886-1930) y en el espacio que cubre el proyecto. A ésto, poco más o menos, se refiere una declaración de Esteban Montejo en el Cimarrón, realmente interesante, en el debate sobre el problema determinante del "¿Quién ha luchado, entonces, en la guerra de la independencia, o bien, cómo fue de alto el porcentaje de ex-esclavos y afrocubanos entre los Libertadores"? Montejo habla en el tono del más profundo convencimiento: "Yo sé que el noventa y cinco por ciento de la raza negra hizo la guerra"10.

En el análisis de las fuentes contemporáneas, los colaboradores del proyecto se habían enfrentado a un lenguaje histórico, que en la mayoría de las ocasiones, era difícil de entender. En el Cimarrón éste parecía ser el lenguaje comprensible, aunque en verdad, no estaba claro hasta qué punto se había corrompido el discurso literario de Montejo, si a través de sus experiencias posteriores o a través de la modificación que hiciera Barnet ${ }^{11}$.

También en la tarea específica de aclarar las situaciones de trabajo en el campo y las estructuras de la propiedad, la lectura del $\mathrm{Ci}$ marrón parecía ofrecer, ante todo, un punto de referencia con respecto al hecho de que Montejo representó precisamente el ejemplo clásico de un anterior exclavo, más tarde el de un cimarrón y por último el de un veterano de la guerra de la independencia privado por los americanos de los frutos de sus contiendas, que después de 1898 tuvo que subsistir de nuevo como agricultor negro y labrador.

De nuevo hay que resaltar que las lecturas del libro han supuesto siempre un recurso de ayuda y por lo tanto, un trasfondo literario que sin embargo, no le concedió apenas importancia científica, o cuando más, ésta fue mínima. El caso es que el Cimarrón era ilustrativo, pero en definitiva, estaba muerto, pues incluso los expertos cubanos admiten que Barnet sólo había inventado a Montejo y que

10 M. BARNET [5], 1967, p. 156.

11 Barnet se ha pronunciado a menudo sobre el problema del idioma, hasta ahora para confirmar que en el Cimarrón (y en otros libros de Barnet) el protagonista sería "el idioma", ver: BARNET, Mesa redonda sobre el testimonio en Revolución y Cultura, nos. 133-134, La Habana 1983, p. 27; y La fuente viva, La Habana 1983, p. 52.

R. I., $1998, \mathrm{n}^{\circ} 212$ 
un auténtico personaje histórico con este nombre no habría podido existir nunca. El mismo Barnet ha favorecido esta presunción con sus afirmaciones en las tres "novelas testimonios" (Biografia de un Cimarrón, La canción de Rachel und Gallego; a las que se añade también $L a$ vida real) y en la desindividualización del idioma ${ }^{12}$.

Más sorprendidos nos quedamos nosotros, cuando investigando intensivamente los protocolos notariales de los archivos de la provincia de Cienfuegos, Santa Clara y en la prensa contemporánea, nos tropezamos reiteradamente con los nombres de "Esteban Montejo y Mera" o simplemente "Esteban Montejo". En un primer momento fue algo enojador - "desde luego no puede ser", "tiene que tratarse de otro" - también continuamos persiguiendo esta pista y nos encontramos con documentos históricos y fuentes primarias que convencían del todo sobre la existencia de un hombre llamado "Esteban Montejo y Mera", en la región de Cruces, Lajas, Palmira y Cienfuegos, desde 1904 hasta 1912. Quien conozca un poco los escollos de la investigación genealógica en una sociedad postesclavista, sabrá que ésto ya es mucho.

El Montejo literario dice en la presentación que hace de sí mismo en el Cimarrón. "Mi primer apellido es Montejo, por mi madre que era una esclava de origen francés. El segundo es Mera. Pero ése casi nadie lo sabe ... El verdadero era Mesa. Lo que sucedió fue que en el archivo me lo cambiaron y lo dejé así, como yo quería tener dos apellidos como los demás para que no me dijeran 'hijo de manigua', me colgué ése y ¡cataplum!"13.

Tras algunas reflexiones y después de buscar otras formas o portadores del nombre de "Montejo" en la lista de veteranos del ejército cubano (Ejército Libertador Cubano; E.L.C.) ${ }^{14}$ y en las nó-

12 Barnet ha detallado recientemente, por primera vez el proceso de creación de el Cimarrón; vea: BARNET, Para llegar a Esteban Montejo ..., [5], passim.

13 M. BARNET [5], p. 16. Barnet cuenta en su trabajo más reciente que en el registro bautismal de Ingenio Flor de Sagua los nombres de los padres de Esteban Montejo aparecen como "Gincongo" (el padre) y "Susana Lucumí" (la madre); ver: BARNET, Para llegar a Esteban Montejo ...,[5], p. 30. De hecho se duda de que en este registro bautismal aparezca Esteban Montejo con los nombres completos de "Esteban Montejo y Mera", como en el mismo lugar afirma BARNET; veáse también la nota siguiente.

14 Conocido como "Indice" con aprox. 74000 nombres de veteranos. Los portadores del nombre "Montejo", registrados en la lista oficial en unidades, en las cuales también ha hecho su servicio el Cimarrón, son extremadamente pocos, para ser exactos concretamente dos, ninguno de los cuales tiene como segundo apellido "Mera". Esteban Montejo y Mera 
minas de ex-combatientes del mismo en la "Gaceta Oficial de la República de Cuba"15 de 1903, nos convencimos de que debía tratarse del verdadero histórico Montejo. ¿Se trataba entoces de la "figura literaria fantástica" de Esteban Montejo en documentos históricos "verdaderos"? ¿Había el Cimarrón vuelto a nacer?

Por el momento se habrían citado los documentos más importantes. El primer grupo de fuentes en el que aparece Montejo lo constituyen las listas de veteranos de la guerra de la independencia del año 1903, las cuales documentan el derecho a la remuneración de sus servicios y los contratos de crédito sobre la suma a pagar, del año 1904.

En la Gaceta Oficial de República de Cuba aparece magníficamente claro escrito: "Montejo Mera, Esteban ... Soldado 982.00"16. Montejo se refiere en su relato exactamente a esta canti$\mathrm{dad}^{17}$. Reproducimos aquí, casi íntegramente, y debido a su importacia como documento, el contrato de crédito proveniente del Archivo Provincial de Cienfuegos que dice:

“...Debito y obligación:

En el pueblo de Santa Isabel de las Lajas, Cabecera del Término de su nombre, partido judicial de Cienfuegos, a veinte y siete de Febrero de mil novecientos cuatro: ante mí Domingo Valdés Losada - Notario Público de este distrito, con residencia en Cruces, previamente requerido para este acto y presente los testigos que se dirán, comparecen: Otorgantes:

\footnotetext{
aparece tal vez en forma corrompida bajo el nombre de "Morejón Mesa, Estéban ... [padres] Alfredo, Emilia ... soldado 3-12-1895" en la Segunda Brigada de la Segunda Division ("Brigada de Cienfuegos"; no. 41463). Rebecca Scott, Orlando García Martínez y el autor estamos inclinados a pensar que debe ser Esteban Montejo, aunque queda la duda, de porqué Montejo le contó a Barnet que su padre se llamó "Nazario" (M. BARNET [5], p. 13], veáse: Índice Alfabético y Defunciones del Ejército Libertador de Cuba. Datos compilados y ordenados por el Inspector General del Ejército Libertador Mayor General Carlos ROLOFF y MiALOFSKY, ayudado del Jefe del Despacho, Comandante de Estado Mayor Gerardo FORREST, Editado oficialmente por disposición del General Leonard WoOD, Gobernador Militar de Cuba, Habana, Imprenta de Rambla y Bouza, Obispo 35, 1901, p. 589. La forma del nombre "Montero", con la que hay verdaderamente muchos, aparece en el "Indice" como tal, claramente reconocible.

15 En las que aparece "Esteban Montejo y Mera"; veáse: Gaceta Oficial de Cuba, Apéndice al N-42, La Habana, martes 18 de agosto de 1903, p. 312.

16 Idem.

17 M. BARNET , [5], p. 123.
}

R. I., $1998, \mathrm{n}^{\circ} 212$ 
De una parte Don Esteban Montejo y Mera, vecino de Cruces, natural de Sagua la Grande, soltero, mayor de edad y agricultor.

Y de otra parte Don Andrés María González y Mora [ $\left.{ }^{18}\right]$, de esta naturalidad y vecindario, casado, mayor de edad y comerciante; que comparece con el carácter de gerente administrador de la sociedad mercantil que gira en este pueblo bajo la razón de González y Compañía, según consta de la escritura de su constitución otorgada ante mí y en mi Notaría con fecha diez y ocho del actual que será oportunamente inscrita en el Registro Mercantil de este Partido. Capacidad [...] Exposición: Primero: Que el Sor. Montejo y Mera, recibe en este acto de la sociedad de González y Compañía la suma líquida de ciento seis pesos, moneda americana, a presencia del Notario autorizante que de ello da fé y de los testigos instrumentales de que le otorga el mas competente recibo y formal carta de pago.

Segundo: Que en su consecuencia y confesándose como se confiesa el Sor. Montejo y Mera líquido y legítimo deudor de los sres. González y Compañía por la expuesta suma de ciento seis pesos moneda americana que es en deberle, se compromete a devolverla a su acreedora, ó a quienes sus derechos representen, sin premio ni interés alguno, tan pronto haga efectivo sus haberes devengados como miembro del Ejército Libertador de Cuba, facultando y confiriendo a dicha sociedad el mas amplio poder para que en su nombre recoja el abonaré que de dichos haberes ha de entregarle el Gobierno de Cuba y para que los cobre en su vía, descontándole su importe la suma adeudada y devolviéndole el remanente que resulte en su favor: consignando que sus alcances, según aparece de la liquidación publicada en la Gaceta de la República, ascienden a novecientos ochenta y dos pesos.

Aceptación

Tercero: El Sor. González y Mora por su parte acepta esta escritura a favor de la sociedad que representa y la compromete a devolver al deudor el remanente que resulte en su favor, deducido el importe de su deuda, en la misma forma en que verifique el pago el Gobierno de Cuba.

Competencia

Cuarto: Los comparecientes designan la ciudad de Cienfuegos como lugar para todas las diligencias [...] y firma el Sor. González y

18 Andrés María González y Mora, desde el 1 de julio de 1900 Primer Teniente Alcalde de Lajas; comerciante de origen español. 
Mora y por Don Esteban Montejo expone no saber lo hace a sus ruegos uno de los testigos Don Eduardo Guzmán y Macías y Don Joaquín Bosch y Sánchez, de este vecindario y hábiles para serlo [...].

Como testigo y a ruego de Esteban Montejo y Mera

Eduardo Guzmán

González y Ca. Joaquín Bosch Domingo V. Losada"19

Ante todo es significativo para nuestro contexto, el hecho de que Montejo por mediación del testigo Eduardo Guzmán y Macías recibiera prestado, en este primer contrato de crédito, 106 pesos de un comerciante español. Esteban Montejo figura otras dos veces, entre los meses de marzo y abril de 1904 en las actas notariales con sumas de dinero relativamente insignificantes, que sin duda, debieron ser muchísimo dinero para un trabajador del campo. Cerca de los 100 pesos: "En el pueblo de Santa Isabel de las Lajas ... D[on]. Esteban Montejo y Mera, vecino de Cruces, natural de Sagua, soltero, mayor y agricultor ...100 pesos en moneda americana" 20 .

En abril de 1904 se trató de nuevo de 224 pesos que le fueron prestados por Eduardo Guzmán ${ }^{21}$. En efecto Montejo aparece inscrito en el documento como vecino de Lajas y según los dos contratos precedentes, como agricultor: "En el pueblo de Santa Isabel de las Lajas ... D[on]. Esteban Montejo y Mera, vecino de Lajas, natural Sagua, soltero, mayor y agricultor ... recibe nuevamente la otra suma líquida de 224 pesos moneda americana"22. La suma asciende en total a 430 pesos. Montejo, a quien correspondían en realidad 982 pesos de "haberes" por su largo tiempo de servicio como ex-soldado, había dejado en manos de sus prestamistas más de la mitad porque necesitaba el dinero sin dilación. La denominación de "agricultor" en los contratos significa que Montejo había dejado de ser un mero labrador, sino que como agricultor intentaba cultivar su propia parcela de tierra y para ello necesitaba dinero. Como se desprende de

\footnotetext{
19 APC, Protocolo Domingo Váldes Losada, tomo 8 (Enero y Febrero de 1904), escritura 148, 27 de febrero de 1904, folio (f) 555r-556v.

20 Ibidem, tomo 9 (marzo de 1904), escritura $\mathrm{n}^{\circ} 201,3$ de marzo de 1904, f. 683r-684v.

21 Guzmán aparece en el contrato de crédito arriba mencionado como testigo y persona que firma para Montejo, es decir, en una posición de confianza.

22 APC, Protocolo Domingo Váldes Losada, tomo 10 (Abril-Mayo de 1904), escritura $n^{\circ} 437,16$ de abril de 1904, f. 1304r-1305v.
} 
esta denominación y contrariamente a la suposición de que Montejo hubiera sido siempre un pobre agricultor, éste llegó a ser entre 1902 y 1904 un (pequeño) propietario, aunque no hemos encontrado todavía el protocolo notarial que así lo atestigüe. Una vez más en un artículo de 1983, una autora escribe en Cuba y la cita debe figurar aquí para la opinión general: "Esclavo primero, pasó a ser obrero agrícola a raíz de la abolición, condición que mantuvo toda su vida ..."23.

También es importante señalar que el testigo y más tarde el mismo prestamista, Eduardo Guzmán y Macías, alcalde de Lajas desde 1902 hasta 1905, aparecen en este documento, respecto al ex-mambí Montejo, en una posición especial de confianza. Las actas notariales no muestran sólo las referencias monetarias, sino también redes clientelares $^{24}$.

El segundo complejo de fuentes comprende los periódicos del año 1912.

En la investigación sobre la participación de negros en la tristemente célebre "guerra de razas" de 1912, dimos con las siguientes citas en el periódico "La Correspondencia" de Cienfuegos:

"MAS REBELDES

Muchos de los indivíduos cuya captura se logra, al prestar declaración en el juzgado, descubren los nombres de otros compañeros que se hallan en armas.

De esta manera ha podido declararse la rebeldía de algunos cabecillas y rebeldes, contra los cuales una vez que se logre su captura, habrá de seguirse también el procedimiento por rebelión.

Ayer fueron declarados rebeldes y dispuesta a la prisión de los siguientes alzados: Esteban Montejo, Domingo Mora, Saturnino Benítez, Cándido Martínez y Benito Cantillo [debe decir: Canutillo M.Z.]."25

\footnotetext{
23 Diana IZnAGa BeIRA, "La novela testimonio de Miguel Barnet: Apuntes sobre una trilogía" en Islas. Revista de la Universidad Central de Las Villas, 83, enero-abril 1986, p. 130.

24 Michael ZeUSKE, Estructuras regionales, movilización afrocubana y "raza" en un hinterland cubano: Cienfuegos entre colonia y república (1895-1912), Mainz 1998 (en prensa).

${ }^{25}$ La Correspondencia, Cienfuegos, mayo 27 de 1912, p. 5 (agradezco a Grisel Ramos Maceda el apoyo en la búsqueda y el trabajo de copiar todas las alusiones a la "guerra de razas", a Esteban Montejo y a Simeón Armenteros en los periódicos La Correspondencia y El Comercio de Cienfuegos).
} 
En otro artículo se titula:

"LOS REBELDES HASTA HOY

... La lista completa de los indivíduos que han sido declarados rebeldes y circulados para que se proceda a su captura, comprende los siguientes individuos:

Simeón Armenteros, Juan Morales, Manuel Labrado (a) Lico; Arcadio y Tomás Benítez, Alejandro Pérez; Felipe Acea (a) Caoba; Secundino y Doroteo Acea; Esteban Torriente; Luis Campos; Máximo Montalvo; Manuel Madruga; Ricardo Cabrera; Esteban Montejo; Domingo Mora; Saturnino Benítez; Cándido Martínez; Benito Canutillo."26

Todos los arrestados y acusados eran negros o mulatos, además de ser, una parte de ellos, antiguos oficiales o soldados de la Guerra de la Independencia.

¿Qué significan estos documentos relativos a la biografía de Miguel Barnet? En primer lugar se prefiere pensar que no mucho porque ninguno de los hechos o acontecimientos de cuya, relación surgen los documentos arriba mencionados, aparecen reflejados en la biografía de Barnet.

Montejo tenía en la época del interrogatorio 103 años ${ }^{27}$. Por consiguiente, aproximadamente en 1900 tenía más o menos 40 años. En el siglo XIX había vivido algo más de un tercio de los 100 años, sin embargo los otros 60 en el siglo XX. Aunque el estudio de Barnet presenta en el Cimarrón únicamente acontecimientos comprendidos hasta 1900 aproximadamente y alusiones que pueden datarse hasta 1909. Dicho de forma maliciosa, Barnet le ha quitado, por lo menos 50 años o al menos no los ha recreado (¿todavía?) para la gloria póstuma de la vida literaria. ¿Podría ser que tal vez Barnet no haya considerado esta parte de la biografía de Montejo precisamente como muy loable y haya dejado sencillamente fuera los fragmentos de la narración del anterior Cimarrón por criterios literarios de su ciclo de novelas testimonios? En efecto, si se lee más exactamente se acaba poniendo de manifiesto que, algunas manifestaciones interesantes aunque generales de Montejo después de 1900, se recogen en el $\mathrm{Ci}$ -

26 Idem.

27 Montejo señala como día de su cumpleaños el 26 de diciembre de 1860, día de San Esteban, ver: M. BARNET [5], p. 13. En total hay que considerar sus testimonios del todo fiables.

R. I., $1998, \mathrm{n}^{\circ} 212$ 
marrón. Sobre todo en los pasajes en los que Montejo da su opinión sobre los problemas raciales. El fragmento más importante del texto es aquél en el que habla sobre la participación de los negros en la lucha por la independencia y el trato de los veteranos negros después de $1899^{28}$. Sin embargo el estudio de Barnet evitó, después del año 1900 prácticamente toda clase de vínculos con cualquier suceso determinado identificable de la historia política. En el ejemplo de los personajes históricos y en las notas a pie de página comentadas, se pone de manifiesto claramente el procedimiento seguido en el estudio. En el fragmento que comprende el período de tiempo posterior a 1899 se menciona a los políticos de color, Martín Morúa Delgado y Generoso Campos Marquetti, así como a Antonio Maceo y Máximo Gómez. En cualquier caso sólo el oficial de color Campos Marquetti tiene una nota a pie de página ${ }^{29}$, la cual a decir verdad, dentro del contexto en el que él aparece en la narración de Montejo, reúne dos afirmaciones que no concuerdan de ninguna manera y que vienen a ser mensajes secretos sobre las actividades de Campos Marquetti en el 1912, annus horribilis: "Generoso Campos Marquetti, representante a la Cámara del Partido Liberal en 1912. Secundó a Martín Morúa Delgado en la ley propuesta y definida por éste que prohibiera la existencia en Cuba de partidos racistas" 30 . El jefe del partido liberal, José Miguel Gómez, fue en esta época presidente de Cuba. La ley mencionada es la "Enmienda Morúa" (1910), incluida en la Constitución cubana de 1901. Ésta incluye un fundamento legal con el objeto de prohibir la composición de partidos "basándose en una raza".

Sin embargo Montejo señala en su narración con seguridad tanto a Morúa como a Campos Marquetti en una connotación positiva, con respecto al hecho de que hubieran sido los únicos que se hubieran ocupado de ello después del final de la guerra, que "dieron algunos puestos en el gobierno a los negros". Con ésto se hubieran opuesto a la tendencia general de"... que los negros se quedaron en la calle" y al mismo tiempo a alejar a los "libertadores negros" 31 de los frutos de la victoria.

\footnotetext{
28 Idem, pp. 156-160.

29 En la edición de 1967 es el número 21; vea: Idem, p. 156; la enumeración de las notas a pie de página es distinta en otras ediciones.

30 Idem.

31 Todas las citas según: el libro de M. BARNET [5] 1967,p. 193s.
} 
El limitado punto de vista que se documenta en el estudio de Barnet comprende la ideología de la revolución cubana posterior a 1959, con el fin de borrar de la memoria colectiva a la República "neocolonial" (1902-1959) con su racismo y volver directamente a las "mejores tradiciones" de Martí.

Con ayuda de un breve y limitado análisis de los documentos anteriormente presentados, intentaremos responder a la pregunta de cuál es ahora, entonces, el "verdadero Montejo".

El origen de los contratos de Esteban Montejo está en relación con los llamados "Haberes" del Ejército Libertador Cubano. Cuando los americanos de E.E.U.U ocuparon el país (1898-99), su primera preocupación fue el desarmar a los cerca de 40.000 combatientes mambises y el disolver al E.L.C. que habían luchado contra España desde 1895 hasta 1898. Ésto se consiguió con la ayuda de Máximo Gómez y la promesa de pago por los servicios prestados a la patria. Primeramente pagaron a los mambises una suma de 75 dólares por la entrega de las armas ("licenciamiento"). El pago principal, la llamada "paga del Ejército Libertador Cubano", se convirtió, de hecho, bajo el gobierno del primer presidente de la República de Cuba, Tomás Estrada Palma, en deuda de Estado ("haberes"). A pesar de esta formulación, el pago se demoró. Además el tema se instrumentalizó políticamente. Incluso los criterios de adjudicación fueron durante algún tiempo dudosos. Aunque los ex-mambises, ahora veteranos, necesitaban dinero urgentemente puesto que la agricultura en el largo tiempo que duró la guerra se había diezmado. Apenas había puestos de trabajo en las ciudades. Se crearon sociedades para la compra, a bajo valor, de títulos de derecho. Una ola de especulaciones sacudió Cuba. ${ }^{32}$. Los especuladores eran, sobre todo, antiguos jefes militares, es decir, altos mandos del disuelto E.L.C. que compraron los derechos a sus anteriores subordinados. Los comerciantes -en muchos casos españoles- solían conceder el capital.

Del contrato y de lo que se menciona en los protocolos notariales resulta evidente que Montejo sólo pudo obtener estos créditos por mediación de Eduardo Guzmán y Macías. Guzmán había fundado, junto con el que fuera por muchos años secretario del Ayuntamiento de Lajas y político autonomista Agustín Cruz y Cruz, una so-

32 Rafael MARTínez ORTIZ, Cuba, los primeros años de su independencia, 2 vols., $\mathrm{Pa}$ ris, Tercera (3a. edición), 1929, II, p. 54.

R. I., $1998, \mathrm{n}^{\circ} 212$ 
ciedad para la concesión de créditos. Eduardo Guzmán había sido capitán del antiguo E.L.C. en la división que estaba al mando de José de Jesús Monteagudo en el Cuarto Cuerpo bajo el Mayor General, José Miguel Gómez. Guzmán era un terrateniente importante y en este tiempo un conocido "cacique político" de la región situada en las inmediaciones de Cruces y Lajas ${ }^{33}$. "Organizaba y dirigía a los rebeldes de su barrio ... un hombre peligroso cuando se trata de organizar escándalo": así lo describen los informes del servicio secreto durante la segunda ocupación americana ${ }^{34}$. Guzmán mantenía estrechas relaciones con el grupo de actores políticos agrupados en torno al ya mencionado José Miguel Gómez. De este grupo de liberales salieron políticos que con posterioridad figuraron entre los hombres más poderosos de Cuba. José Miguel, como lo llamaban sus amigos, fue primeramente Gobernador Provincial de Las Villas y de 1909 a 1913 segundo presidente de Cuba. Alfredo Zayas fue presidente desde 1921 hasta 1925. A este grupo pertenecía también Gerardo Machado, el que más tarde fuera asímismo presidente y dictador. Fue precisamente en el tiempo que se ocultó en la biografía de Barnet, cuando comenzaron sus carreras políticas. Eduardo Guzmán, como General, dirigió en 1906 el levantamiento de los liberales contra el gobierno conservador de Estrada Palma en la región de Lajas-Cruces ("guerrita de agosto"). En 1904, Esteban Montejo tenía con este hombre, según consta, relaciones clientelares que para él, por supuesto, se podían tachar de relaciones importantes con un "amigo" 35 .

Es decir, volviendo a la visión histórica del libro Biografía de un Cimarrón, Montejo era "amigo" de "politicastros" y ¡estaba vinculado a políticos como José Miguel Gómez y Gerardo Machado a través de Don Eduardo! Después de 1959 la historiografía oficial les trató, de hecho, como persona inexistente al primero y como mero dictador al segundo, olvidando que ellos, en la Guerra de Indepen-

33 Michael ZeusKe, "Die diskrete Macht der Sklaven: Zur politischen Partizipation von Afrokubanern während des kubanischen Unabhängigkeitskrieges und der ersten Jahre der Republik (1895-1908) - eine regionale Perspektive en Idem (ed.), Nach der Sklaverei,"Comparativ, Leipzig, 1997, vol. 7/1, pp. 32-98; 95.

34 USNA, Record Group (RG) 395, Records of U.S. Army Overseas Operation and Commands, 1898-1942, Army of Cuban Pacifiction, Military Intelligence Division, Entry (E) 1008, File (F) 79, Item (I) 30.

35 Michael ZEUSKE, [33], pp. 79-90. 
dencia, fueron importantísimos colaboradores de Máximo Gómez en los difíciles años de 1897/98.

Algo parecido es válido para el segundo complejo de fuentes, en las que se menciona a Montejo para las comunicaciones de prensa del año 1912. La "guerra de razas" de este año es un dramático y traumático acontecimiento político de la historia cubana más reciente, el cual hasta ahora apenas se había tratado ${ }^{36}$.

Los independentistas cubanos agrupados en torno a José Martí y Aıtonio Maceo hicieron su aparición con el programa le Cuba "con todos y para el bien de todos". La igualdad de las "razas", fue uno de los temas importantes, si no el esencial de este programa. Después de 1902 los cubanos negros exigieron el cumplimiento de este programa. En 1908 se constituyó el primer partido "negro" en la historia mundial, el "Partido Independiente de Color" (PIC), bajo la dirección de soldados veteranos para luchar por la igualdad de razas en la arena política. En 1910 se prohibió en Cuba, con la mencionada ley Morúa, la constitución de partidos, bajo la que cayó el PIC. En el 1912, año de elecciones, se trasladaron los dirigentes de este partido (prohibido), Evaristo Estenoz y Pedro Ivonet a la provincia del este, Oriente, con el fin de convocar un levantamiento, siguiendo la tradición de las guerras de independencia y con ello ejercer presión política para la readmisión de su partido. Este levantamiento fue seguido en todo el país por los simpatizantes del PIC. En las otras provincias fueron, en realidad, tan pocos que los "alzamientos" producidos, como por ejemplo en la zona de Cienfuegos, pudieron ser rápidamente sofocados. Montejo y su jefe, el famoso Simeón Armenteros, fueron a parar a la cárcel ${ }^{37}$. Sin embargo, en Oriente la designación de las Fuerzas Armadas de la República, recayó en General José de Jesús Monteagudo, un hombre del grupo de actores alrededor de José Miguel Gómez, el cual —recordamos- fue presi-

36 Aline HELG, Our Rightful Share. The Afro-Cuban Struggle for Equality 1886-1912, Chapel Hill and London The University of North Carolina Press 1995; Serafin PoRTUONDO LiNARES, El Partido Independiente de Color, La Habana 1950; Rafael FoRMOSElle, Política y color: la guerrita de 1912, Montevideo 1974; Tomás FERNÁNDEZ RoBAINA, El negro en Cuba 1902-1958. Apuntes para una historia de la lucha contra la discriminación racial, La Habana 1990.

37 Alexandra BRoNFMAN, "Clientelism and Chaos in Cienfuegos, 1912", ponencia inédita, The First Cuban Research Institute's Conference on Cuba and Cuban-American Studies (citado con el permiso de la autora).

R. I., $1998, \mathrm{n}^{\circ} 212$ 
dente en este período. En el transcurso de las distintas razzias en los montes de Oriente por parte del ejército repúblicano se asesinaron, por así decir, a varios miles de negros ${ }^{38}$.

Pero, ¿qué tienen que ver estos acontecimientos y la posible participación de Montejo en ellos con el discurso histórico de comienzos de los sesenta en Cuba, el cual también fue seguido de forma evidente por Barnet? La revolución cubana de 1959 había escrito en sus banderas, más radicalmente incluso que los actores independentistas de la Guerra de la Independencia de 1895-1898, la igualdad y armonía racial. La "guerra de razas" de 1912 tenía que ser válida, desde el punto de vista oficial de fraternidad de todos los hombres - y según la postura anterior y contemporánea de la historiografíano sólo como atavismo en lo que respecta a una parte de los oficiales blancos y veteranos de la Guerra de la Independencia, como un arrebato inexplicable de los cubanos por matar, en general, sino también como "racismo", es decir, desde la perspectiva de la mentalidad cubana integralista, como "separación" de los dirigentes del PIC del programa de armonía racial y del martianismo.

Esta perspectiva comprende, más o menos, la opinión de los medios de comunicación contemporáneos y el recuerdo histórico gene$\mathrm{ral}^{39}$. El consenso nacional surgido de la guerra se convirtió después de 1898 y sobre todo después de 1902 en componente de la mitología patriótica controlada por las élites blancas, que influyó con más intensidad a las masas, a través del culto a Martí, aunque al mismo tiempo las visiones sobre la igualdad que tenían los negros, los cuales habían crecido durante la Guerra de Independencia y los catorce hasta 1912, se fueron "olvidando"40. Ada Ferrer y Rebecca Scott han podido comprobar al respecto que algunos de los prominentes insurrectos negros ya en la guerra del 95 habían formulado una visión sobre la igualdad

38 Thomas ORUM, The Politics of Color: The Racial Dimension of Cuban Politics during the Early Republican Years, 1900-1912, Ph. Diss, New York University 1975; Louis A. PÉREZ Jr., Louis A., "Politics, Peasants, and People of Color: The 1912 'Race War' in Cuba Reconsidered" en Hispanic American Historical Review 66 (August 1986), pp. 509539, Lords of the Mountain: Social Banditry and Peasant Protes in Cuba, 1878-1918, Pittsburgh: University of Pittsburgh Press 1989; otra versión radicalmente distinta: HELG, [32], pp. 193-225.

39 Idem, pp. 228-248.

40 Rebeca ScotT, "Raza, clase y acción colectiva en Cuba, 1895-1912: formación de alianzas interraciales en el mundo de la caña", en OP.CIT., Revista del Centro de Investigaciones Históricas, núm. 9, San Juan, 1997, pp. 131-157, en especial pp. 131-136. 
que iba más allá de la igualdad concedida por los blancos ${ }^{41}$. Los dirigentes del PIC intentaron realizar esta visión sobre la igualdad. Cuando se les negó la participación en las votaciones, optaron por la imitación de la tradicional insurrección que debía llevarse a cabo en la República contra las nuevas elites que, por otra parte, devolvieron la pelota e hicieron uso de su legitimidad para reprochar a los dirigentes del PIC la sediciosa falta de lealtad.

Desde el punto de vista de entonces, Montejo no hubiera podido participar en un acontecimiento tal, incluso la misma "guerra de razas" y desde esta perspectiva anterior jtampoco habría tenido lugar! Así que lo más fácil resultó guardar silencio sobre el asunto o borrarlo de la cinta. Evidentemente y para el mismo Montejo, el recuerdo de su participación en las batallas de 1912 fue desagradable. En su narración, los acontecimientos sólo aparecen en relación con Martín Morúa Delgado. Montejo habla sobre la "guerra de razas" como un acontecimiento que sólo ha tenido lugar en Oriente: Morúa "... hasta provocó la revuelta de negros de Alto Songo"42.

¿A qué juicio debe llegar el historiador que conoce estas fuentes $y$, con qué expectativa, de manera que en el transcurso del proyecto arriba mencionado o de otras investigaciones surjan posiblemente, todavía más menciones de Montejo?

En primer lugar se puede afirmar que cincuenta años de "silencio" y el no recoger estas informaciones en la biografía del Cimarrón son, sin lugar a dudas, demasiados. En segundo lugar: incluso los acontecimientos o los complejos, en cuyo contexto se sitúa lo mencionado por Montejo y que muy probablemente son omitidos por Barnet, alumbran de forma significativa el problema de las "razas" en Cuba.

Las dudas sobre la metodología utilizada en el procedimiento de Barnet como historiador - ¡no como literato! - se confirman, en cierto modo, si no es que aumentan todavía más. Por otra parte, habla también el peso del discurso histórico contemporaneo, bajo el que Barnet compiló la biografía, en cierto modo por las observaciones anteriores. "Die Eule fliegt erst in der Dämmerung" (Hegel: "La

41 Idem; Ada Ferrer, To Make a Free Nation: Race and the Struggle for Cuban Independence, 1868-1898, Ph. D.., University of Michigan, 1995 (no publicado).

42 M. BARNET [5], p. 150. En BARNET, La canción de Rachel, Barcelona, Editorial Estela, 1970, pp. 58-60, Montejo defiende el programa del PIC, no tanto el alzamiento mismo, como sostiene William Luis; véase: William LuIS, Literary Bondage. Slavery in Cuban Narrative, Austin, The University of Texas Press, 1990, pp. 214-215. 
lechuza siempre vuela primero al anocher") y a los historiadores nacidos después y con mejores condiciones de investigación y otras perspectivas, les resulta muy fácil denunciar públicamente los supuestos "errores" de las generaciones anteriores. Así que, en ningún caso, debe menoscabarse el auténtico mérito de Barnet por haber dado a conocer a un público mundial y haber despertado la conciencia sobre el problema de los anteriores esclavos de Cuba. Sin embargo Miguel Barnet hubiera debido quizás escribir un nuevo epílogo en la edición o traducción siguiente del Cimarrón o un segundo tomo del Cimarrón.

En tercer lugar, y lo que parece ser el resultado más importante del presente ensayo, las declaraciones de Montejo en el Cimarrón, las cuales se revalorizan mucho, incluso sin que sepamos si sus recuerdos sobre los hechos confirmados a través de las citas documentales arriba mencionadas se encuentran en las famosas cintas magnetofónicas que Miguel Barnet debe conservar aún en alguna parte.

La biografía y el comportamiento de Esteban Montejo se corresponden con el comportamiento de algunos cientos de anteriores mambises negros o de color que pudieron ser analizados durante el proyecto. El hinterland de Cienfuegos, situada en el interior del país, alrededor de Lajas, Palmira y Cruces fue a comienzos del siglo XX una zona con una gran producción azucarera. Desde la abolicion de la esclavitud atrajo de forma magnética, no sólo a anteriores esclavos y esclavas, sino también a otros muchos agricultores, obreros y pequeños propietarios. En los protocolos notariales del archivo de la provincia de Cienfuegos se identifican, sólo procedentes en las poblaciones de Cruces y Lajas o Palmira escasamente a 400 ciudadanos de color ${ }^{43}$, los cuales intentaron convertir en dinero los títulos legales que tenían en los "Haberes". Invirtieron la mayoría de este dinero en la producción de subsistencia. De los aproximadamente 400 ciudadanos, alrededor de 200 soldados veteranos no habían nacido en la región o se habían afincado allí durante algún tiempo. Procedían de la jurisdicción de Cienfuegos, más alejada, de la misma ciudad, de la provincia Las Villas y de las provincias de Matanzas, La Habana, Oriente e incluso Pinar del Río. Después de 1899 se establecieron en la región de Lajas-Cruces. Como Esteban Montejo,

43 Hemos podido analizar 816 protocolos notariales de antiguos mambises en la región de Lajas-Cruces; 371 de ellos eran afrocubanos; M. ZEUSKE, [33], pp. 65-79. 
intentaron comenzar una nueva vida. Como él, mantuvieron relaciones de clientela con "caciques políticos" blancos o negros de los alrededores. Como a él, les decepcionó la política de los liberales después de 1908, reunidos en torno a José Miguel Gómez, no sólo en lo relativo a la igualdad racial, sino también en relación a su falta de apoyo a los pequeños propietarios y a su rudo comportamiento con respecto a sus reivindicaciones para mejorar las condiciones de vida de los trabajadores del azúcar ${ }^{44}$. Sólo en la jurisdicción de Cienfuegos, aproximadamente cien negros y humbres de color, veteranos, en su mayoría agricultores siguieron en 1912 la misma actitud de Montejo, incluso hombres jóvenes provenientes del oscuro círculo del bandolerismo ${ }^{45}$.

Como en otras provincias de Cuba fuera de Santiago, esta protesta fue, sin embargo, más bien marginal y Montejo como algunos de sus "amigos" de color fueron rápidamente a la cárcel, aunque al poco tiempo eran puestos otra vez en libertad tras la amnistía decretada apresuradamente ${ }^{46}$.

Sin embargo Montejo experimentó en cada caso mucho más que en los años 40 en el siglo XIX, años a los cuales Barnet se restringió en su libro. ¡El cimarrón vive!

Through the analysis of archival documents (notarial protocols) and newspapers from 1912, the author provides a critical reading of the novel-testimony Biografia de un Cimarrón (1966) by Miguel Barnet. In addition, the paper discusses the results of the Cuban Independence War (1895-1898), comparing the literary perspectives of Barnet, Montejo's narration and the discourse of the primary sources.

44 John Dumoulin, "El primer desarrollo del movimiento obrero y la formación del proletariado en el sector azucarero. Cruces 1886-1902", Islas, $n^{\circ} 48$, Cienfuegos, mayojunio 1974, pp. 3-66.

${ }^{45}$ ANC, Secretaría de la Presidencia, leg. 110, $\mathrm{N}^{\circ}$ 2: Expediente referente a los alzamientos de negros, dirigidos por el partido independiente de color, encabezados por Evaristo Estenoz y Pedro Ivonet; fecha: Habana, Santiago de Cuba, Pinar del Río, Guanajay, 17 de junio a 9 de septiembre de 1912, 2 vols.

46 Existen razones verdaderamente plausibles, como Aline Helg únicamente supone (HELG, [32], p. 202, notas 41 y 43), en un primer momento hubo un "agreement" entre los dirigentes del PIC, sobre todo Pedro Ivonet, y José Miguel Gómez, para poder efectuar la reelección de este último. Sin embargo el control sobre el ejército se le fue de las manos a Gómez en 1912 y el "agreement" no funcionó; véase la carta de Pedro Ivonet enviada a José Miguel Gómez desde Santiago de Cuba, 2. febrero de 1910, en: Archivo del Museo de la Ciudad (La Habana), legajo 68, expediente 39, núm. doc. 397,001. 